\title{
Bandwidth Efficient OFDM Transmitter Diversity Techniques
}

\author{
King F. Lee \\ Multimedia Architecture Lab, Motorola Labs, Schaumburg, IL 60196, USA \\ Email:king.lee@motorola.com \\ Douglas B. Williams \\ School of Electrical and Computer Engineering, Georgia Institute of Technology, Atlanta, GA 30332-0250, USA \\ Email:douglas.williams@ece.gatech.edu
}

Received 17 December 2002; Revised 2 September 2003

Space-time block-coded orthogonal frequency division multiplexing (OFDM) transmitter diversity techniques have been shown to be efficient means of achieving near-optimal diversity gain in frequency-selective fading channels. However, these known techniques all require a cyclic prefix to be added to the transmitted symbols, resulting in bandwidth expansion. In this paper, iterative space-time and space-frequency block-coded OFDM transmitter diversity techniques are proposed that exploit spatial diversity to improve spectral efficiency by eliminating the need for a cyclic prefix.

Keywords and phrases: space-time coding, space-frequency coding, transmitter diversity, OFDM, channel estimation, pilot symbols.

\section{INTRODUCTION}

The last decade has witnessed an explosive growth of wireless communications, especially in mobile communications and personal communications services (PCS). With the continuing expansion in both existing and new markets and the introduction of exciting new services such as wireless internet access and multimedia applications, the wireless communications market is expected to continue to grow at a rapid pace. Furthermore, the ever-increasing demand for faster and more reliable services to support new applications has created strong interests in developing high data rate wireless communications systems. With existing and emerging wireless applications, all competing for a limited radio spectrum, the development of high data rate wireless communications systems that are spectrally efficient is especially important.

The main challenge in developing reliable high data rate mobile communications systems is to overcome the detrimental effects of frequency-selective fading in mobile communications channels. A number of space-time coded orthogonal frequency division multiplexing (OFDM) transmitter diversity techniques have recently been proposed for high data rate wireless communications $[1,2,3,4]$. It has been shown in $[3,4]$ that space-time and space-frequency block-coded OFDM (STBC-OFDM and SFBC-OFDM) sys- tems are efficient means of achieving near optimum diversity gain in frequency-selective fading channels. These previously proposed OFDM transmitter diversity systems all require a cyclic prefix to be added to the transmitted symbols to avoid intersymbol interference (ISI) and interchannel interference (ICI) in the OFDM symbols, and the number of cyclic prefix symbols has to be equal to or greater than the order of the wireless channels [5]. The addition of the cyclic prefix causes bandwidth expansion if a desired data rate is to be maintained or a reduction in data rate if the transmission bandwidth is fixed. For many high data rate systems, the addition of a cyclic prefix can cause more than a $15 \%$ bandwidth expansion, which is a very significant loss of a valuable resource [6]. In this paper, we propose iterative space-time and spacefrequency block-coded OFDM (ISTBC-OFDM and ISFBCOFDM) transmitter diversity techniques that do not require a cyclic prefix and, therefore, are more bandwidth efficient than previously proposed systems.

Computer simulations are used extensively to evaluate the performances of the various systems considered in this paper. The COST207 six-ray typical urban (TU) channel power delay profile [7] is used to model the frequencyselective fading channels in all the simulations. Furthermore, for the simulations in Sections 2 and 3, perfect estimates of the channel impulse responses (CIRs) are assumed to be available at the receiver. 


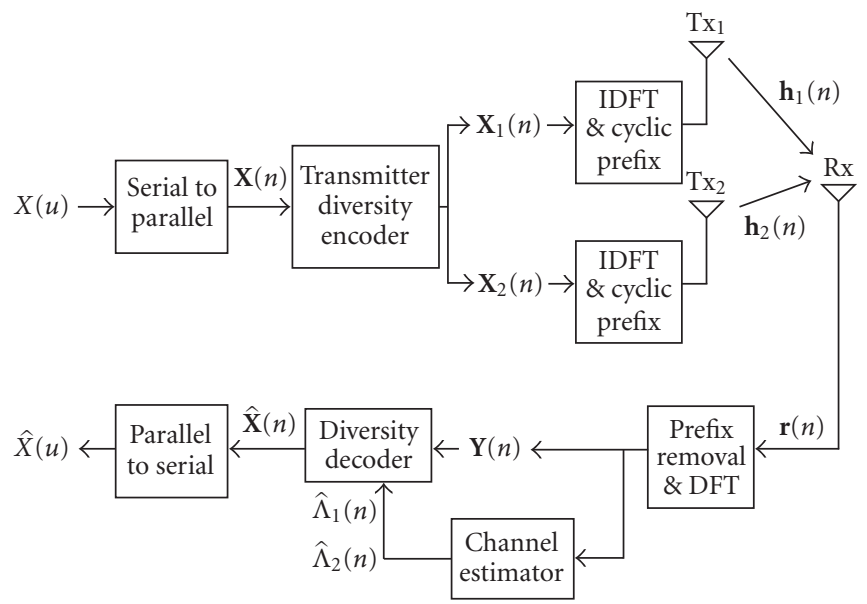

FIGURE 1: Block diagram of a two-branch OFDM transmitter diversity system utilizing a cyclic prefix.

The remainder of the paper is organized as follows. In Section 2, a brief overview of OFDM transmitter diversity systems utilizing a cyclic prefix is provided. Section 3 gives a detailed description of the proposed bandwidth efficient ISTBC-OFDM and ISFBC-OFDM transmitter diversity systems. Section 4 considers channel estimation techniques for OFDM transmitter diversity systems without a cyclic prefix. Finally, Section 5 summarizes the results and outlines possible future research in this area.

\section{OFDM TRANSMITTER DIVERSITY SYSTEMS UTILIZING A CYCLIC PREFIX}

A block diagram of a general two-branch OFDM transmitter diversity system with a cyclic prefix is shown in Figure 1. Let $X(u)$ denote the input serial data symbols with symbol duration $T_{S}$. The serial to parallel converter collects $K$ serial data symbols into a data vector $\mathbf{X}(n)=$ $\left[\begin{array}{llll}X(n, 0) & X(n, 1) & \cdots & X(n, K-1)\end{array}\right]^{T}$, which has a block duration of $K T_{S} .{ }^{1}$ The transmitter diversity encoder codes $\mathbf{X}(n)$ into two vectors $\mathbf{X}_{1}(n)$ and $\mathbf{X}_{2}(n)$ according to an appropriate coding scheme as in $[1,2,3,4]$. The coded vector $\mathbf{X}_{1}(n)$ is modulated by an inverse discrete Fourier transform (IDFT) into an OFDM symbol sequence. A length $G$ cyclic extension is added to the OFDM symbol sequence and the resulting signal is transmitted from the first transmit antenna. Similarly, vector $\mathbf{X}_{2}(n)$ is modulated by an IDFT, cyclically extended, and transmitted from the second transmit antenna. Let $\mathbf{h}_{1}(n)$ denote the CIR between the first transmit antenna and the receiver and let $\mathbf{h}_{2}(n)$ denote the CIR between the second transmit antenna and the receiver. To avoid ISI and ICI, the length of the cyclic extension $G$ is chosen to be greater than or equal to $L$, the maximum order of the CIRs, that is, $G \geq L$ [5]. At the receiver, the received signal vector first has the

\footnotetext{
${ }^{1}$ Throughout the paper, we will use the notation that $A(n, k)$ denotes the $k$ th element of the vector $\mathbf{A}(n)$.
}

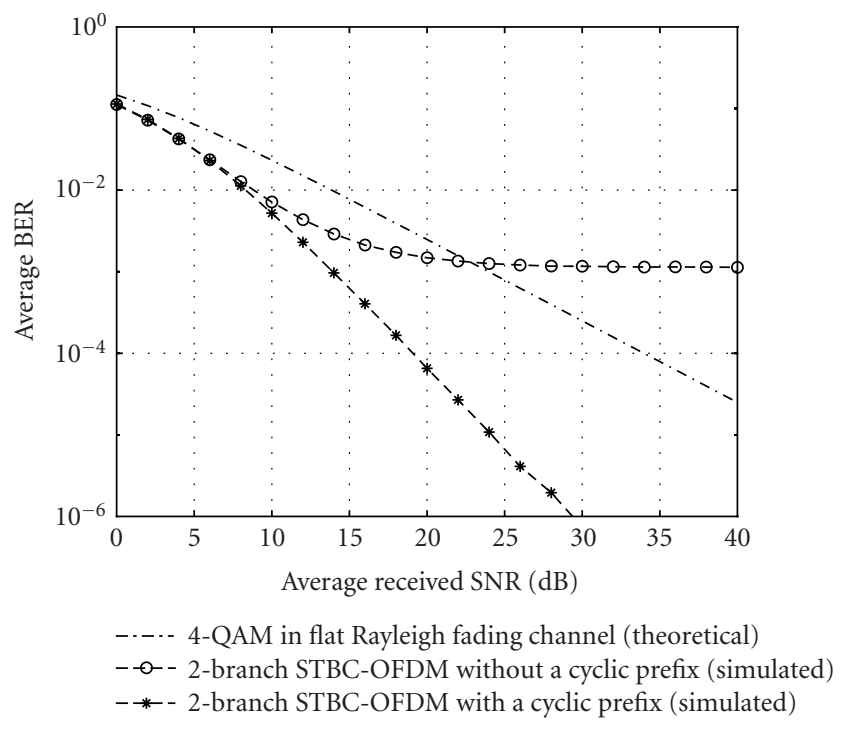

FIGURE 2: Performance of STBC-OFDM without a cyclic prefix in a TU channel with $T_{S}=2^{-20}$ second, $K=32, L=5$, and $f_{D}=10 \mathrm{~Hz}$.

cyclic prefix removed and is then demodulated by a discrete Fourier transform (DFT) to yield the demodulated signal vector $\mathbf{Y}(n)$. Assuming the CIRs remain constant during the entire block interval, the demodulated signal is given by $[3,4]$

$$
\mathbf{Y}(n)=\boldsymbol{\Lambda}_{1}(n) \mathbf{X}_{1}(n)+\boldsymbol{\Lambda}_{2}(n) \mathbf{X}_{2}(n)+\mathbf{Z}(n),
$$

where $\Lambda_{1}(n)$ and $\Lambda_{2}(n)$ are two diagonal matrices whose elements are the DFTs of the respective CIRs and $\mathbf{Z}(n)$ is the DFT of the channel noise. Elements of $\mathbf{Z}(n)$ are generally assumed to be additive white Gaussian noise (AGWN) with variance $\sigma_{Z}^{2}$.

In OFDM systems, the use of a cyclic prefix transforms the linear convolution between the transmitted symbols and the frequency-selective CIR into circular convolution. The IDFT and DFT pair used in the OFDM modulation and demodulation processes then transforms the time-domain circular convolution into simple multiplication in the frequency domain. The net effect is that OFDM with a cyclic prefix transforms the frequency-selective fading channel into multiple perfectly decoupled flat fading subchannels. The OFDM transmitter diversity systems in $[1,2,3,4]$ all rely on this special property of OFDM with a cyclic prefix in the precoding and decoding processes to achieve good diversity performance. Without the cyclic prefix, the convolution between the transmitted symbols and the frequency-selective CIR reverts back to the usual linear convolution, causing ISI and ICI in the OFDM systems. As a result, the underlying OFDM subchannels are no longer decoupled flat fading channels and the diversity performance of STBC-OFDM and SFBC-OFDM transmitter diversity systems is significantly degraded.

For example, Figure 2 shows simulation results of the BER performances for an STBC-OFDM transmitter diversity system in a slow fading channel with maximum Doppler 
frequency $f_{D}=10 \mathrm{~Hz}$, both with and without a cyclic prefix. The example STBC-OFDM system has a block size $K=32$ and channel order $L=5$, requiring a cyclic prefix of length 5 with the resultant bandwidth expansion of $L \div K=15.6 \%$. Figure 2 clearly shows the degradation of the diversity gain for STBC-OFDM without a cyclic prefix. Although not shown here, performances of SFBC-OFDM transmitter diversity systems without a cyclic prefix exhibit similar degradations.

\section{BANDWIDTH EFFICIENT OFDM TRANSMITTER DIVERSITY SYSTEMS}

As described in Section 2 and demonstrated in the example of Figure 2, the performances of STBC-OFDM and SFBCOFDM transmitter diversity systems are significantly degraded without the cyclic prefix. Therefore, in order to eliminate the cyclic prefix requirement for STBC-OFDM and SFBC-OFDM systems, some form of ISI and ICI equalization for these OFDM transmitter diversity systems is needed. A number of equalization techniques have been proposed to reduce the negative effects of ISI and ICI for OFDM systems without a cyclic prefix or when the cyclic prefix is shorter than the channel memory $[8,9,10,11,12]$. Unfortunately, these equalization techniques are highly channel specific, that is, the equalizer coefficients are strong functions of the channel response. With transmitter diversity, as shown in Figure 1, the received signal is the superposition of signals transmitted simultaneously from multiple transmitters and the channel responses between each transmitter and the receiver are generally different. An equalizer that can simultaneously equalize the channel responses from all the transmitters does not exist, in general. Therefore, any equalization technique that is specific to the channel response will not be effective for transmitter diversity systems. However, here a compensation technique that is only "partially" dependent on the channel responses will be shown to be very effective for STBC-OFDM and SFBC-OFDM transmitter diversity systems without a cyclic prefix. The proposed technique, described in detail in the following sections, provides an effective and efficient means of eliminating the need for a cyclic prefix for the STBC-OFDM and SFBC-OFDM transmitter diversity systems, thus eliminating the bandwidth expansion while still achieving very good diversity performance.

The proposed technique extends the tail cancellation and cyclic reconstruction ideas shown in [13] and the iterative technique shown in [14] to STBC-OFDM and SFBC-OFDM transmitter diversity systems. Therefore, the proposed techniques will be referred to as ISTBC-OFDM and ISFBCOFDM transmitter diversity. The ISTBC-OFDM and ISFBCOFDM techniques rely on two key properties of the IDFT and DFT.

(1) The IDFT and DFT pair diagonalizes any circulant matrix. This property is equivalent to the more familiar property of the DFT where circular convolution in the time domain equates to simple multiplication in the frequency domain. This property is the key to transforming a frequency-selective fading channel into multiple completely decoupled flat fading subchannels.

(2) The IDFT and DFT are linear transforms and superposition holds when applied to the received signal in a transmitter diversity system, which is a sum of signals from multiple transmitters. Linearity allows the transforms to operate on the received signal components without any undesirable cross-terms.

The proposed technique is applicable to both STBC-OFDM and SFBC-OFDM transmitter diversity systems. Since the ISFBC-OFDM transmitter diversity algorithm is simpler, the ISFBC-OFDM technique will be described first in Section 3.1 followed by the ISTBC-OFDM algorithm in Section 3.2.

\subsection{ISFBC-OFDM systems}

A block diagram of the ISFBC-OFDM system is shown in Figure 3. Let $\mathbf{X}(n)$ denote the $K \times 1$ vector at the output of the serial to parallel converter at the block instant $n$. The spacefrequency encoder codes $\mathbf{X}(n)$ into vectors $\mathbf{X}_{1}(n)$ and $\mathbf{X}_{2}(n)$ according to the coding scheme for SFBC-OFDM [4]. The SFBC vectors $\mathbf{X}_{1}(n)$ and $\mathbf{X}_{2}(n)$ are modulated by the IDFT into time-domain OFDM signals $\mathbf{x}_{1}(n)$ and $\mathbf{x}_{2}(n)$ and then transmitted through channels with CIRs $\mathbf{h}_{1}(n)$ and $\mathbf{h}_{2}(n)$. Note that no cyclic prefix is added to either $\mathbf{x}_{1}(n)$ or $\mathbf{x}_{2}(n)$, so there is no bandwidth expansion or rate reduction. For presentation simplicity, the additive channel noise will be omitted in the following derivation. In the absence of noise, the received signal vector is given by

$$
\mathbf{r}(n)=\mathbf{x}_{1}(n) * \mathbf{h}_{1}(n)+\mathbf{x}_{2}(n) * \mathbf{h}_{2}(n),
$$

where $*$ denotes linear convolution. Equivalently, the received signal vector can be expressed in terms of convolution matrices of the CIRs and transmitted signal vectors as follows:

$$
\begin{aligned}
\mathbf{r}(n)= & \mathbf{H}_{1,0} \mathbf{x}_{1}(n)+\mathbf{H}_{1,1} \mathbf{x}_{1}(n-1) \\
& +\mathbf{H}_{2,0} \mathbf{x}_{2}(n)+\mathbf{H}_{2,1} \mathbf{x}_{2}(n-1),
\end{aligned}
$$

where the first index in the subscript denotes the spatial dimension and the second index denotes the temporal dimension. The convolution matrices $\mathbf{H}_{m, 0}$ and $\mathbf{H}_{m, 1}$ are defined in terms of the CIRs $\mathbf{h}_{m}(n)$ as follows:

$$
\left[\begin{array}{ccccccc}
h_{m, 0} & 0 & \cdots & \cdots & \cdots & \cdots & 0 \\
h_{m, 1} & h_{m, 0} & 0 & \ddots & \ddots & \ddots & \vdots \\
\vdots & h_{m, 1} & h_{m, 0} & \ddots & \ddots & \ddots & \vdots \\
h_{m, L} & \ddots & \ddots & \ddots & \ddots & \ddots & \vdots \\
0 & \ddots & \ddots & \ddots & h_{m, 0} & 0 & \vdots \\
\vdots & \ddots & \ddots & \ddots & h_{m, 1} & h_{m, 0} & 0 \\
0 & \cdots & 0 & h_{m, L} & \cdots & h_{m, 1} & h_{m, 0}
\end{array}\right],
$$




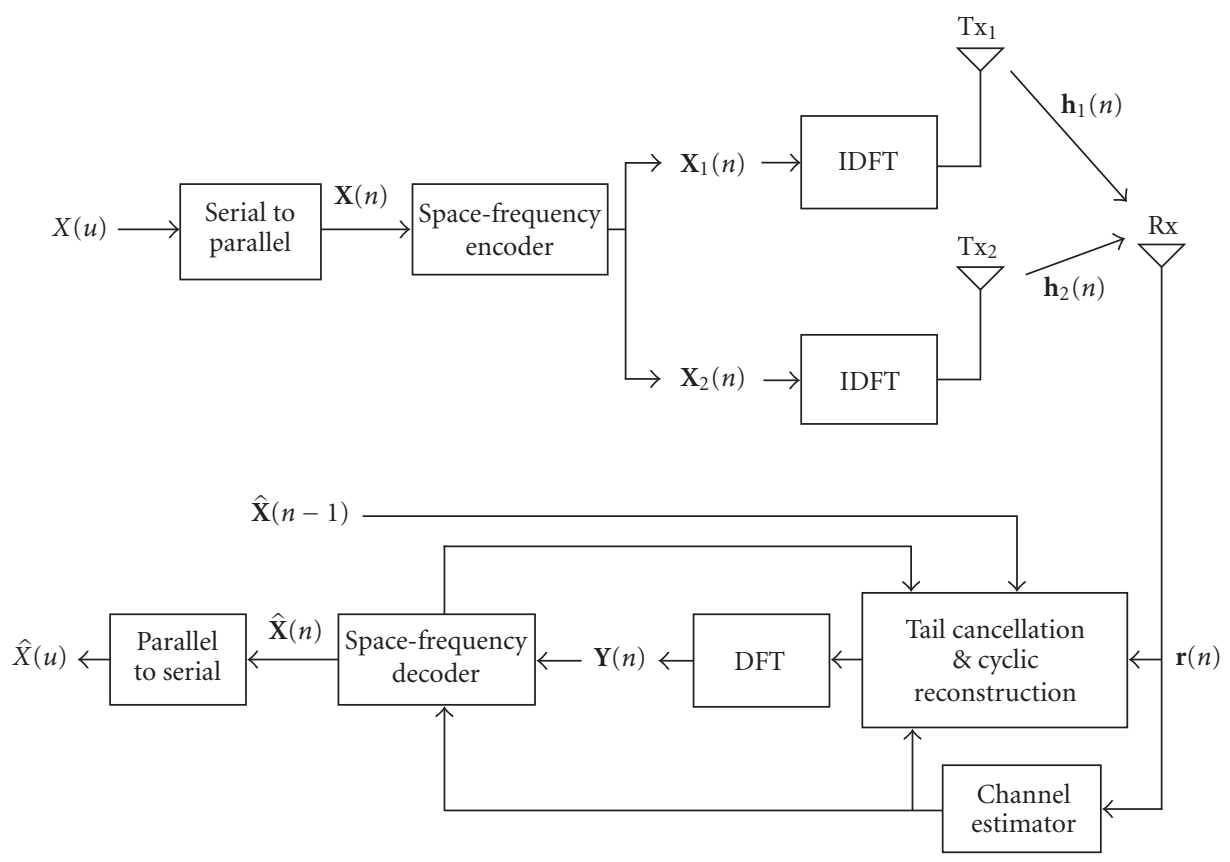

Figure 3: Block diagram of the ISFBC-OFDM transmitter diversity system.

$$
\left[\begin{array}{ccccccc}
0 & \cdots & 0 & h_{m, L} & \cdots & h_{m, 2} & h_{m, 1} \\
\vdots & 0 & \ddots & 0 & \ddots & \ddots & h_{m, 2} \\
\vdots & \ddots & \ddots & \ddots & \ddots & \ddots & \vdots \\
\vdots & \ddots & \ddots & \ddots & \ddots & 0 & h_{m, L} \\
\vdots & \ddots & \ddots & \ddots & \ddots & \ddots & 0 \\
\vdots & \ddots & \ddots & \ddots & \ddots & 0 & \vdots \\
0 & \cdots & \cdots & \cdots & \cdots & \cdots & 0
\end{array}\right],
$$

respectively, where $m=1$ and 2 and the implicit dependency of the time-varying CIRs on the block instant $n$ has been omitted for briefness of presentation. The $\mathbf{H}_{1,1} \mathbf{x}_{1}(n-1)$ and $\mathbf{H}_{2,1} \mathbf{x}_{2}(n-1)$ terms in (3) represent contributions from the previous block that can be eliminated using the previous decision $\hat{\mathbf{X}}(n-1)$ and the estimated channel responses $\widehat{\mathbf{h}}_{m}(n)$ from the channel estimator. Notice that $\mathbf{x}_{1}(n-1)$ and $\mathbf{x}_{2}(n-1)$ are simply the IDFTs of the SFBC $\mathbf{X}(n-1)$, so they can be estimated from $\hat{\mathbf{X}}(n-1)$. Elimination of the contribution from $\mathbf{x}_{1}(n-1)$ and $\mathbf{x}_{2}(n-1)$ is referred to as tail cancellation [13] and can be achieved by

$$
\begin{aligned}
\widetilde{\mathbf{r}}(n) & =\mathbf{r}(n)-\hat{\mathbf{H}}_{1,1} \hat{\mathbf{x}}_{1}(n-1)-\hat{\mathbf{H}}_{2,1} \hat{\mathbf{x}}_{2}(n-1) \\
& \approx \mathbf{H}_{1,0} \mathbf{x}_{1}(n)+\mathbf{H}_{2,0} \mathbf{x}_{2}(n) .
\end{aligned}
$$

On the other hand, the desired received signal for SFBCOFDM transmitter diversity, which has the correct circular convolution (or cyclic) property, has the form

$$
\begin{aligned}
\mathbf{y}(n)= & \mathbf{H}_{1,0} \mathbf{x}_{1}(n)+\mathbf{H}_{1,1} \mathbf{x}_{1}(n) \\
& +\mathbf{H}_{2,0} \mathbf{x}_{2}(n)+\mathbf{H}_{2,1} \mathbf{x}_{2}(n) .
\end{aligned}
$$

Notice that $\left(\mathbf{H}_{1,0}+\mathbf{H}_{1,1}\right)$ is a circulant matrix corresponding to $\mathbf{h}_{1}(n),\left(\mathbf{H}_{2,0}+\mathbf{H}_{2,1}\right)$ is a circulant matrix for $\mathbf{h}_{2}(n)$, and (6) is simply the sum of circular convolutions. The equivalent equation in the frequency domain is

$$
\mathbf{Y}(n)=\Lambda_{1}(n) \mathbf{X}_{1}(n)+\Lambda_{2}(n) \mathbf{X}_{2}(n)
$$

where $\Lambda_{1}(n)$ and $\boldsymbol{\Lambda}_{2}(n)$ are diagonal matrices whose elements are the DFTs of the respective CIRs $\mathbf{h}_{1}(n)$ and $\mathbf{h}_{2}(n)$. The time-domain equation in (6), or equivalently the frequency-domain equation in (7), is the desired ISIand ICI-free flat fading subchannel system we are attempting to achieve. Hence, the goal is to add an estimate of $\mathbf{H}_{1,1} \mathbf{x}_{1}(n)+\mathbf{H}_{2,1} \mathbf{x}_{2}(n)$ to $\tilde{\mathbf{r}}(n)$ to approximate the desired signal $\mathbf{y}(n)$. Adding an estimate of $\mathbf{H}_{1,1} \mathbf{x}_{1}(n)+\mathbf{H}_{2,1} \mathbf{x}_{2}(n)$ to $\tilde{\mathbf{r}}(n)$ amounts to restoring the cyclic property of the SFBC-OFDM system and is referred to as cyclic reconstruction [13]. Since $\mathbf{x}_{1}(n)$ and $\mathbf{x}_{2}(n)$ are functions of the yet-to-be-determined symbol vector $\mathbf{X}(n), \mathbf{x}_{1}(n)$ and $\mathbf{x}_{2}(n)$ are not readily available for the cyclic reconstruction. The iterative approach in [14] is therefore adapted here for the cyclic reconstruction process.

A flow diagram of the ISFBC-OFDM algorithm is shown in Figure 4, and an outline of the algorithm is as follows.

(1) Space-frequency code the previous decision $\hat{\mathbf{X}}(n-1)$ into $\hat{\mathbf{X}}_{1}(n-1)$ and $\hat{\mathbf{X}}_{2}(n-1)$ and modulate with an IDFT to form $\hat{\mathbf{x}}_{1}(n-1)$ and $\hat{\mathbf{x}}_{2}(n-1)$. Tail cancellation is then performed on the received signal vector $\mathbf{r}(n)$ to form $\widetilde{\mathbf{r}}(n)$ as in (5). Initialize iteration number $i$ to zero.

(2) Demodulate $\widetilde{\mathbf{r}}(n)$ with a DFT and decode with the space-frequency decoder and decision device to form 


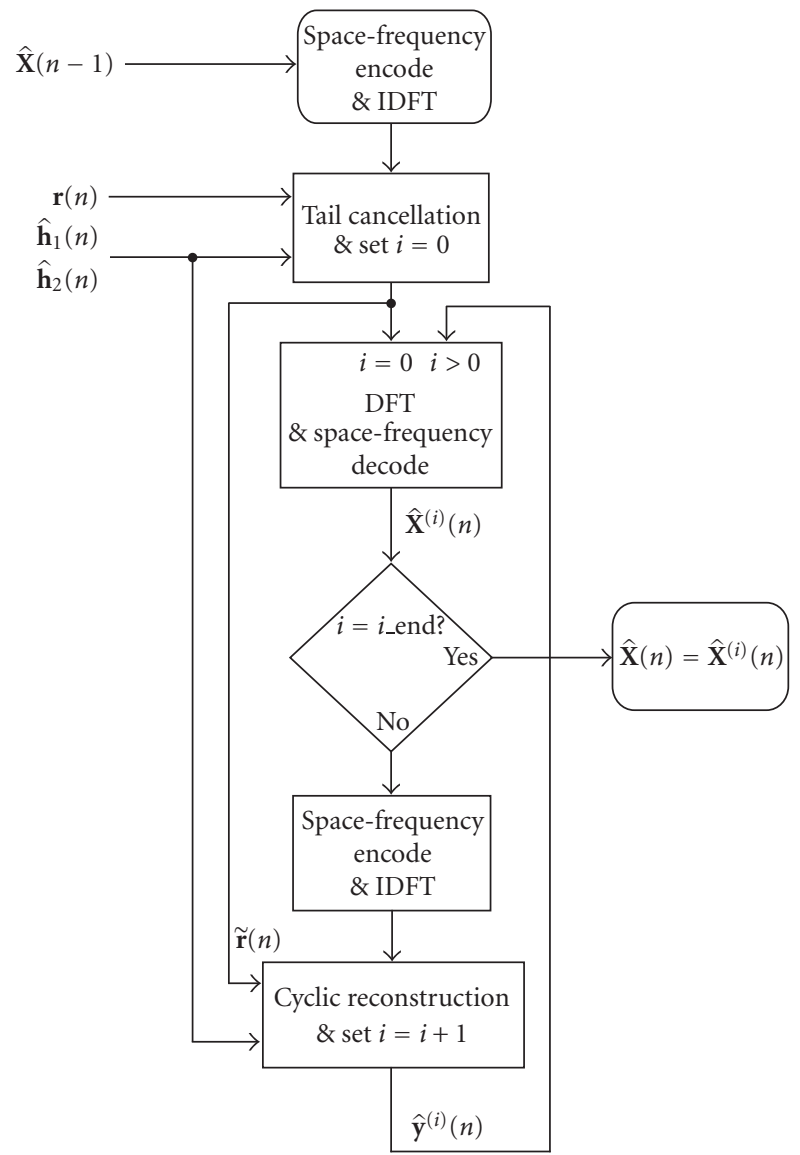

FIGURE 4: Flow diagram of the ISFBC-OFDM transmitter diversity algorithm.

the estimate $\hat{\mathbf{X}}^{(0)}(n) .^{2}$

(3) Space-frequency code $\hat{\mathbf{X}}^{(i)}(n)$ into $\hat{\mathbf{X}}_{1}^{(i)}(n)$ and $\hat{\mathbf{X}}_{2}^{(i)}(n)$ and modulate with an IDFT to form $\widehat{\mathbf{x}}_{1}^{(i)}(n)$ and $\hat{\mathbf{x}}_{2}^{(i)}(n)$.

(4) Form the cyclic reconstructed signal as

$$
\mathbf{y}^{(i)}(n)=\widetilde{\mathbf{r}}(n)+\widehat{\mathbf{H}}_{1,1} \widehat{\mathbf{x}}_{1}^{(i)}(n)+\widehat{\mathbf{H}}_{2,1} \widehat{\mathbf{x}}_{2}^{(i)}(n)
$$

and increment the iteration number to $i=i+1$.

(5) An updated decision on $\mathbf{X}(n)$ can then be obtained from $\mathbf{y}^{(i)}(n)$ with a DFT, space-frequency decoding, and passing through the decision device to yield the updated decision $\widehat{\mathbf{X}}^{(i)}(n)$.

(6) Repeat steps 3-5 for a predetermined number of times to obtain the final decision $\widehat{\mathbf{X}}(n)$.

Simulation results for a two-branch ISFBC-OFDM transmitter diversity system at various iterations $(i=0,1$, and 2$)$ are shown in Figure 5. For the simulations in Sections 3.1 and 3.2 , perfect estimates of the CIRs are assumed to be available at the receiver. Simulation results in Figure 5 show that

\footnotetext{
${ }^{2}$ The parenthesized superscript will be used to denote the iteration number, for example, $\widehat{\mathbf{X}}^{(1)}(n)$ is the estimate of $\widehat{\mathbf{X}}(n)$ after the first iteration.
}

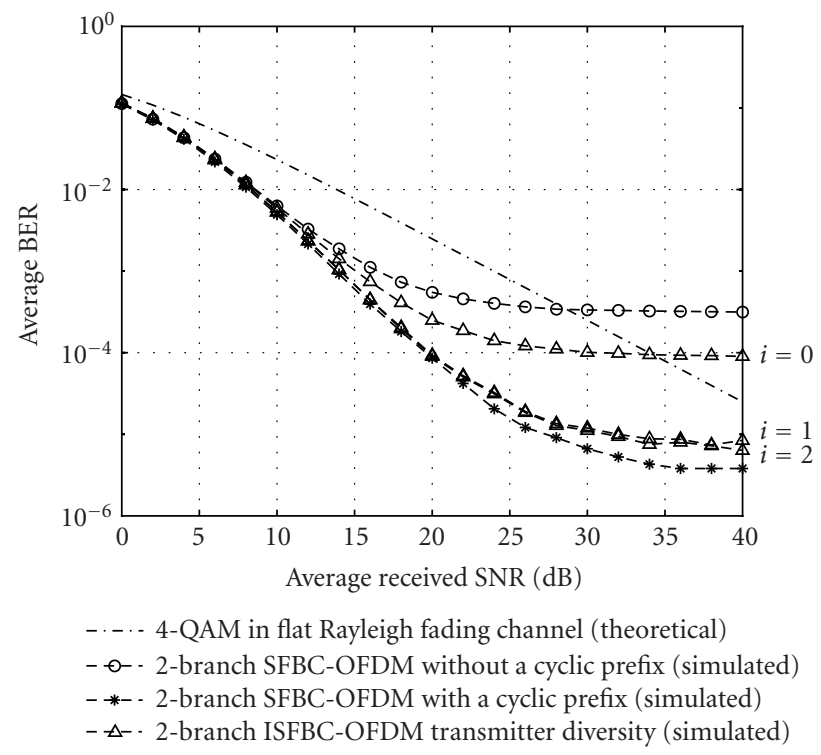

FIGURE 5: Performance of ISFBC-OFDM transmitter diversity in a TU channel with $T_{S}=2^{-21}$ second, $K=256, L=10$, and $f_{D}=$ $20 \mathrm{~Hz}$. Iteration number is indicated for $i=0,1$, and 2 .

the ISFBC-OFDM transmitter diversity system provides significantly better performance over that of the SFBC-OFDM without a cyclic prefix. For this example, the performance of the ISFBC-OFDM system approaches that of the SFBCOFDM with a cyclic prefix within just one to two iterations.

\subsection{ISTBC-OFDM systems}

The ISTBC-OFDM transmitter diversity system will be described next. A block diagram of the ISTBC-OFDM system is shown in Figure 6. For the two-branch STBC-OFDM system, the diversity encoding and decoding are performed on two consecutive data blocks over two block instants [3]. The space-time encoder codes $\mathbf{X}(n)$ and $\mathbf{X}(n+1)$ into two vector pairs $\left[\mathbf{X}_{1}(n), \mathbf{X}_{1}(n+1)\right]$ and $\left[\mathbf{X}_{2}(n), \mathbf{X}_{2}(n+1)\right]$ using the coding scheme for STBC-OFDM, where $n$ is incremented by two for every two block instants. The STBC vectors $\mathbf{X}_{1}(n), \mathbf{X}_{1}(n+1), \mathbf{X}_{2}(n)$, and $\mathbf{X}_{2}(n+1)$ are first modulated by an IDFT into time-domain OFDM signals $\mathbf{x}_{1}(n)$, $\mathbf{x}_{1}(n+1), \mathbf{x}_{2}(n)$, and $\mathbf{x}_{2}(n+1)$ and then transmitted through channels with CIRs $\mathbf{h}_{1}(n)$ and $\mathbf{h}_{2}(n)$. In the absence of noise, the received signals during the two corresponding block instants are given by

$$
\begin{aligned}
\mathbf{r}(n)= & \mathbf{H}_{1,0} \mathbf{x}_{1}(n)+\mathbf{H}_{1,1} \mathbf{x}_{1}(n-1) \\
& +\mathbf{H}_{2,0} \mathbf{x}_{2}(n)+\mathbf{H}_{2,1} \mathbf{x}_{2}(n-1), \\
\mathbf{r}(n+1)= & \mathbf{H}_{1,0} \mathbf{x}_{1}(n+1)+\mathbf{H}_{1,1} \mathbf{x}_{1}(n) \\
& +\mathbf{H}_{2,0} \mathbf{x}_{2}(n+1)+\mathbf{H}_{2,1} \mathbf{x}_{2}(n) .
\end{aligned}
$$

Here, the desired signals with the correct cyclic property are

$$
\begin{aligned}
\mathbf{y}(n)= & \mathbf{H}_{1,0} \mathbf{x}_{1}(n)+\mathbf{H}_{1,1} \mathbf{x}_{1}(n) \\
& +\mathbf{H}_{2,0} \mathbf{x}_{2}(n)+\mathbf{H}_{2,1} \mathbf{x}_{2}(n), \\
\mathbf{y}(n+1)= & \mathbf{H}_{1,0} \mathbf{x}_{1}(n+1)+\mathbf{H}_{1,1} \mathbf{x}_{1}(n+1) \\
& +\mathbf{H}_{2,0} \mathbf{x}_{2}(n+1)+\mathbf{H}_{2,1} \mathbf{x}_{2}(n+1) .
\end{aligned}
$$




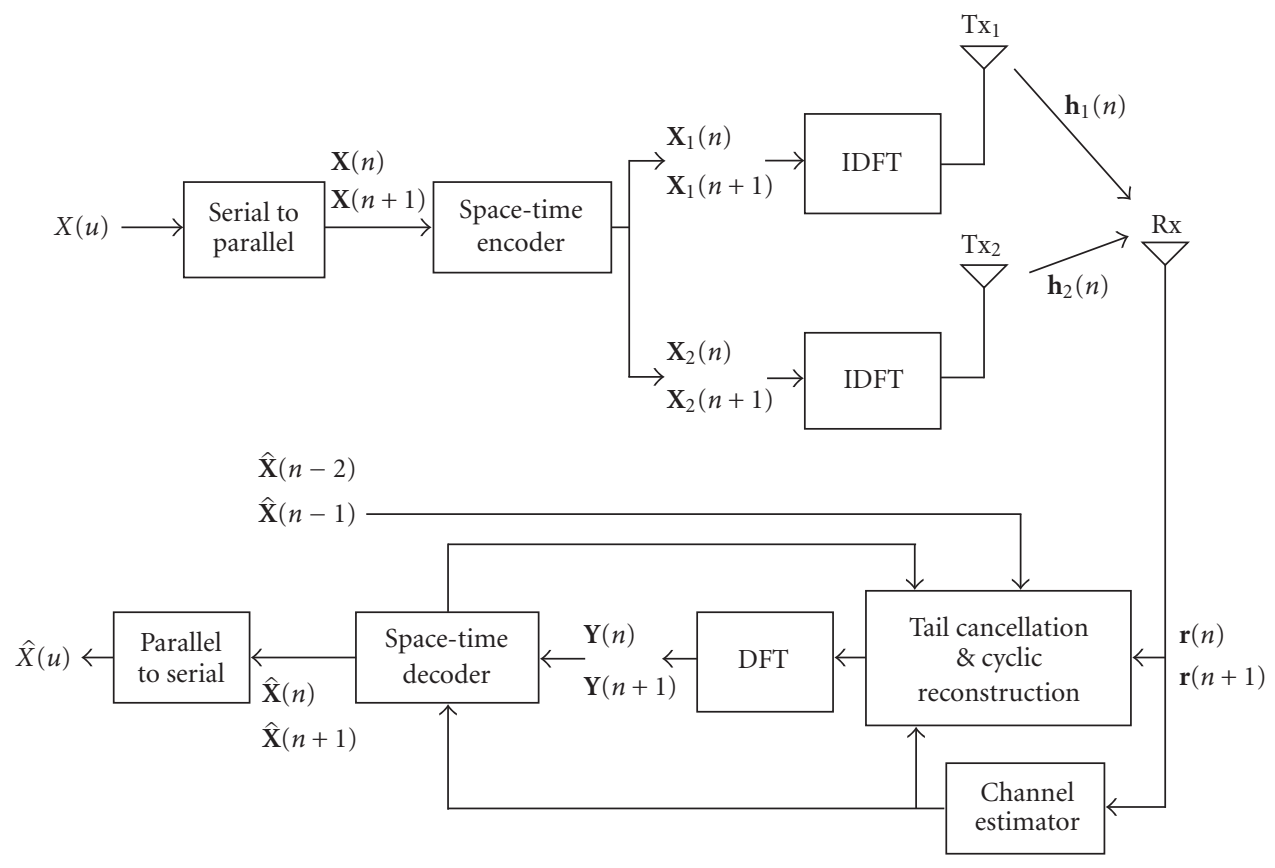

FIGURE 6: Block diagram of the ISTBC-OFDM transmitter diversity system.

Tail cancellation can be performed on $\mathbf{r}(n)$ with the previous decisions $\hat{\mathbf{X}}(n-2)$ and $\hat{\mathbf{X}}(n-1)$ as follows:

$$
\widetilde{\mathbf{r}}(n)=\mathbf{r}(n)-\widehat{\mathbf{H}}_{1,1} \widehat{\mathbf{x}}_{1}(n-1)-\hat{\mathbf{H}}_{2,1} \widehat{\mathbf{x}}_{2}(n-1),
$$

where $\widehat{\mathbf{x}}_{1}(n-1)$ and $\widehat{\mathbf{x}}_{2}(n-1)$ are the IDFTs of the STBC $\hat{\mathbf{X}}(n-2)$ and $\hat{\mathbf{X}}(n-1)$. Cyclic reconstruction of $\mathbf{y}^{(i)}(n)$ can be done similarly to the steps in the ISFBC-OFDM algorithm except that the space-time block coding is used instead. Tail cancellation for $\mathbf{r}(n+1)$, however, requires $\hat{\mathbf{X}}(n)$ and $\hat{\mathbf{X}}(n+1)$, which are still to be determined. Therefore, the ISTBC-OFDM algorithm requires some modifications from that of the ISFBC-OFDM. Recall that with the ISFBC-OFDM algorithm, the tail cancellation step is performed once in the beginning and only the cyclic reconstruction is updated iteratively. For ISTBC-OFDM, both the tail cancellation and cyclic reconstruction for $\mathbf{y}^{(i)}(n+1)$ have to be done through iterative updates.

A flow diagram for the ISTBC-OFDM algorithm is shown in Figure 7 and an outline of the algorithm is as follows.

(1) Space-time code the previous decisions $\hat{\mathbf{X}}(n-1)$ and $\hat{\mathbf{X}}(n-2)$ and modulate with an IDFT to form $\hat{\mathbf{x}}_{1}(n-1)$ and $\hat{\mathbf{x}}_{2}(n-1)$. Tail cancellation is then performed on the received signal vector $\mathbf{r}(n)$ to form $\widetilde{\mathbf{r}}(n)$ as in (11). Initialize iteration number $i$ to zero.

(2) Demodulate $\widetilde{\mathbf{r}}(n)$ and $\mathbf{r}(n+1)$ with a DFT and decode using the space-time decoder and decision device to form the estimates $\hat{\mathbf{X}}^{(0)}(n)$ and $\hat{\mathbf{X}}^{(0)}(n+1)$.

(3) Space-time code $\hat{\mathbf{X}}^{(i)}(n)$ and $\hat{\mathbf{X}}^{(i)}(n+1)$ and modulate with an IDFT to form $\widehat{\mathbf{x}}_{1}^{(i)}(n), \widehat{\mathbf{x}}_{1}^{(i)}(n+1), \widehat{\mathbf{x}}_{2}^{(i)}(n)$, and $\widehat{\mathbf{x}}_{2}^{(i)}(n+1)$.
(4) Form the cyclic reconstructed signal $\mathbf{y}^{(i)}(n)$ as in (8).

(5) Perform tail cancellation and cyclic reconstruction on $\mathbf{r}(n+1)$ as follows:

$$
\begin{aligned}
\mathbf{y}^{(i)}(n+1)= & \mathbf{r}(n+1)-\hat{\mathbf{H}}_{1,1} \widehat{\mathbf{x}}_{1}^{(i)}(n)-\hat{\mathbf{H}}_{2,1} \widehat{\mathbf{x}}_{2}^{(i)}(n) \\
& +\widehat{\mathbf{H}}_{1,1} \widehat{\mathbf{x}}_{1}^{(i)}(n+1)+\widehat{\mathbf{H}}_{2,1} \widehat{\mathbf{x}}_{2}^{(i)}(n+1)
\end{aligned}
$$

and increment the iteration number as $i=i+1$.

(6) An updated decision on $\mathbf{X}(n)$ and $\mathbf{X}(n+1)$ can then be obtained from $\mathbf{y}^{(i)}(n)$ and $\mathbf{y}^{(i)}(n+1)$ by performing a DFT, space-time decoding, and passing through the decision device to yield the updated decisions $\hat{\mathbf{X}}^{(i)}(n)$ and $\hat{\mathbf{X}}^{(i)}(n+1)$.

(7) Repeat steps 3-6 for a predetermined number of times to obtain the final decisions $\hat{\mathbf{X}}(n)$ and $\hat{\mathbf{X}}(n+1)$.

Simulation results for a two-branch ISTBC-OFDM transmitter diversity system at various iterations $(i=0,1,2$, and 3) are shown in Figure 8. Simulation results show that the ISTBC-OFDM transmitter diversity system provides significant improvement over STBC-OFDM without a cyclic prefix. For this particular example, ISTBC-OFDM provides over $12 \mathrm{~dB}$ of diversity gain at a BER of $10^{-4}$ and lowers the error floor from $10^{-3}$ to $2 \times 10^{-5}$.

\subsection{Computational complexity}

As compared to STBC-OFDM and SFBC-OFDM systems, ISTBC-OFDM and ISFBC-OFDM systems require additional computations to combat the ISI and ICI caused by the lack of a cyclic prefix. The additional complexity depends on several system parameters, such as the block size $K$, the number of iterations $i$, and the channel order $L$. In 


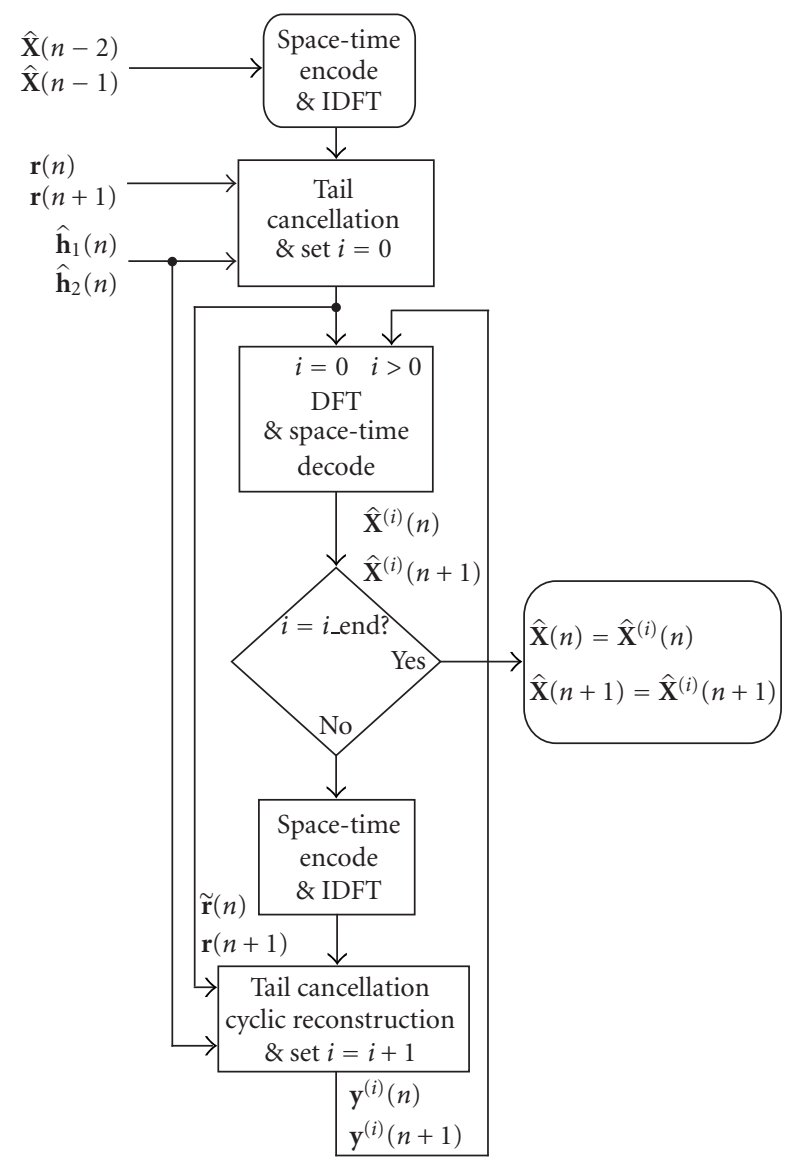

FIGURE 7: Flow diagram of the ISTBC-OFDM transmitter diversity algorithm.

this section, the computational complexities of the ISTBCOFDM and ISFBC-OFDM algorithms are considered. First, notice that the space-time and space-frequency block encodings involve only minor reindexing, negation, and conjugation, which is negation of the imaginary part. These operations have essentially zero cost and, therefore, will not be counted in the computational load of the algorithms. The computational complexity of the ISTBC-OFDM and ISFBCOFDM algorithms for each OFDM block, that is, every $K$ data symbols, is summarized in Tables 1 and 2, respectively. Since the block size $K$ is usually much larger than the channel order $L$, the convolution matrices used in the tail cancellation and cyclic reconstruction steps are generally sparse. Therefore, the multiplication operations for the tail cancellation and cyclic reconstruction have only minor impact on the computational loads. As shown in Tables 1 and 2, the ISTBC-OFDM and ISFBC-OFDM algorithms have about the same computational loads, especially when the number of iterations is large, and most of the computational complexity is in the DFTs. To lessen the computational load, the block size $K$ can be chosen to be a power of two so that a highly efficient FFT algorithm, which requires only approximately $(K / 2) \log _{2} K$ multiplications and $K \log _{2} K$ additions [15], can be used.

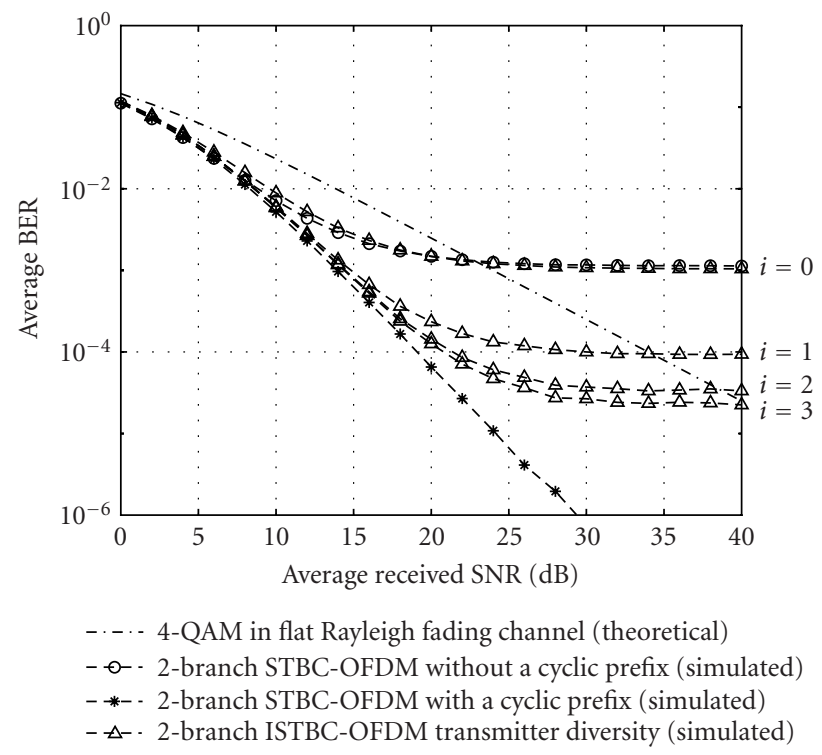

Figure 8: Performance of ISTBC-OFDM transmitter diversity in a TU channel with $T_{S}=2^{-20}$ second, $K=32, L=5$, and $f_{D}=10 \mathrm{~Hz}$. Iteration number is indicated for $i=0,1,2$, and 3 .

Compared with other equalization techniques for OFDM systems without a sufficient cyclic prefix $[8,9,10,11,12]$, which often have a computational complexity of $\mathcal{O}\left(K^{3}\right)$ for a block size of $K$ [16], the proposed ISTBC-OFDM and ISFBCOFDM algorithms have significantly lower computational loads. More importantly, as mentioned at the beginning of this section, none of the techniques shown in $[8,9,10,11,12]$ is applicable to multiple transmitter systems. Therefore, the proposed ISTBC-OFDM and ISFBC-OFDM algorithms are not only efficient but also the only techniques known to the authors that are applicable to OFDM transmitter diversity systems without a cyclic prefix.

Although ISTBC-OFDM and ISFBC-OFDM transmitter diversity systems incur additional computational complexity beyond that required by STBC-OFDM and SFBC-OFDM systems, the added computational loads allow for significant improvement in bandwidth efficiency. It is important to note that radio spectrum is a limited resource while the computation powers of signal processors continue to double about every eighteen months [17]. Therefore, tradeoffs between bandwidth efficiency and reasonable increases in computational complexity will likely continue to be in favor of the bandwidth efficient approaches.

\section{CHANNEL ESTIMATION FOR ISTBC-OFDM AND ISFBC-OFDM SYSTEMS}

It has been shown in previous sections that the ISTBCOFDM and ISFBC-OFDM transmitter diversity techniques are effective and efficient means of achieving good diversity gain in frequency-selective fading channels without requiring the use of a cyclic prefix. For these systems, knowledge of the channel parameters is required at the receivers 
TABLE 1: Computational complexity of the ISTBC-OFDM algorithm.

\begin{tabular}{l|c|c|c}
\hline & DFTs & Multiplications & Additions \\
\hline Tail cancellation & 1 & $\frac{L(L+1)}{2}$ & $\frac{L(L+1)}{2}$ \\
Cyclic reconstruction (per iteration) & 3 & $\frac{3 L(L+1)}{2}+2 K$ & $\frac{3 L(L+1)}{2}+K$ \\
Total complexity for $i$ iterations & & $\frac{3 i+1}{2} K \log _{2} K+2 i K+\frac{3 i+1}{2} L(L+1)$ & $(3 i+1) K \log _{2} K+i K+\frac{3 i+1}{2} L(L+1)$ \\
\hline
\end{tabular}

TABLE 2: Computational complexity of the ISFBC-OFDM algorithm.

\begin{tabular}{l|c|c|c}
\hline & DFTs & Multiplications & Additions \\
\hline Tail cancellation & 2 & $L(L+1)$ & $L(L+1)$ \\
Cyclic reconstruction (per iteration) & 3 & $L(L+1)+2 K$ & $L(L+1)+K$ \\
Total complexity for $i$ iterations & & $\frac{3 i+2}{2} K \log _{2} K+2 i K+(i+1) L(L+1)$ & $(3 i+2) K \log _{2} K+i K+(i+1) L(L+1)$ \\
\hline
\end{tabular}

${ }^{\ddagger}$ Assuming $K$ is a power of two and each FFT requires $(K / 2) \log _{2} K$ multiplications and $K \log _{2} K$ additions.

for tail cancellation, cyclic reconstruction, and decoding. All the impressive diversity gain results shown in Figures 5 and 8 were achieved under the assumption that perfect channel information was available at the receiver. In practice, the receiver has to estimate the channel information and the channel estimation process is usually far from perfect. Channel estimation techniques for conventional OFDM systems have been studied extensively by many researchers $[18,19,20,21,22,23,24]$. However, channel estimation for OFDM systems with transmitter diversity has seen only limited development so far. Channel estimation for transmitter diversity systems is generally complicated by the fact that signals transmitted simultaneously from multiple antennas become interference for each other during the channel estimation process. In this section, we study channel estimation techniques that are compatible with OFDM transmitter diversity systems without a cyclic prefix and are thus applicable to ISTBC-OFDM and ISFBC-OFDM systems.

In [25], a decision-directed MMSE channel estimator for OFDM systems with transmitter diversity was proposed. The main drawback of the MMSE channel estimation approach is the high computational complexity required to update the channel estimates during the data transmission mode. More importantly, the channel estimator in [25] requires the subchannels to be completely decoupled. In the absence of a cyclic prefix of sufficient length, the subchannels are no longer decoupled and the performance of the estimator is significantly degraded. Therefore, a different channel estimation approach is needed for the ISTBC-OFDM and ISFBCOFDM systems. In this section, we consider an extension of the multirate pilot-symbol-assisted (PSA) channel estimation technique proposed in [26] to OFDM transmitter diversity systems without a cyclic prefix, making it suitable for the ISTBC-OFDM and ISFBC-OFDM systems.

The lack of a cyclic prefix in ISTBC-OFDM and ISFBCOFDM systems presents a particular challenge to the channel estimation process. Without a sufficiently long cyclic prefix, the subchannels of these OFDM systems are distorted by ISI and ICI. Thus, the desirable decoupled relationship in (1), which both the decision-directed MMSE channel estimator in [25] and the PSA channel estimator in [26] depend on, is no longer valid. Therefore, neither the decision-directed MMSE channel estimator in [25] nor the PSA channel estimator in [26] is directly applicable to the ISTBC-OFDM and ISFBC-OFDM systems. With the decision-directed approach, in addition to minimizing the interference among the multiple transmitted signals, the channel estimator would also have to eliminate the ISI and ICI caused by the lack of the cyclic prefix during the data transmission mode. Hence, any decision-directed approach is unlikely to yield an effective channel estimator for the ISTBC-OFDM and ISFBC-OFDM systems. On the other hand, with the PSA channel estimator, the ISI and ICI caused by the lack of the cyclic prefix only need to be eliminated during the pilot mode, which is generally an easier problem to be solved. Therefore, we propose a modification to the PSA channel estimator in [26], making it suitable for OFDM transmitter diversity systems without a cyclic prefix.

First, an interesting property of any length $K$ sequence $s(m)$ with only even harmonics, that is, all the odd frequency bins are zero, is that the sequence $s(m)$ is periodic in $K / 2$. That is, $s(m)=s(m+K / 2)$ for $0 \leq m \leq K / 2-1$. The first half of the sequence is in effect the cyclic extension of the second half of the sequence and, therefore, can be used just like a length $K / 2$ guard interval for the second half of the sequence [14]. The PSA channel estimator developed in [26] can be extended to work with OFDM transmitter diversity systems without a cyclic prefix by using pilot sequences with the above cyclic property.

Define a length $K$ chirp sequence as follows:

$$
C(k)=e^{j\left(\pi k^{2} / K\right)}, \quad 0 \leq k \leq K-1 .
$$

Let $\mathrm{PS}_{m}(n, k)$ denote the $k$ th tone of the pilot symbol 
transmitted from the $m$ th transmit antenna during the block instant $n$. The pilot symbols are constructed as follows:

$$
\begin{aligned}
& \mathrm{PS}_{m}(n, k+2(m-1)) \\
& \quad= \begin{cases}(-1)^{m} \sqrt{M} C(k+2(m-1)) & \text { if }(k)_{2 M}=0, \\
0 & \text { otherwise, }\end{cases}
\end{aligned}
$$

where $C(k)$ is the chirp sequence as defined in (13), $M$ is the number of transmitters, $(k)_{2 M}$ denotes $k$ modulo $(2 M)$, $1 \leq m \leq M, 0 \leq k \leq K-1$, and $1 \leq m+k \leq K$. Figure 9 shows the pilot symbol patterns for a typical twobranch OFDM transmitter diversity system without a cyclic prefix. Notice that the pilot symbols in Figure 9 satisfy the following properties.

(1) The pilot symbols transmitted from different transmitters occupy different frequency bins. This property enables the avoidance of interference among pilot symbols from different transmitters and is the same property as that implemented for the channel estimator in [26].

(2) The pilot symbols transmitted from the same transmitter have only nonzero values on even subcarriers. This property ensures that the time-domain pilot sequence is periodic in $K / 2$ so that the first half of the sequence can serve as the guard interval for the second half of the sequence.

At the receiver, the last $K / 2$ samples of the received signal vector $\mathbf{r}_{\mathrm{PS}}(n)$ are assigned to the vector $\mathbf{y}(n)$ as follows:

$$
y(n, k)= \begin{cases}r_{\mathrm{PS}}\left(n, k+\frac{K}{2}\right) & \text { for } 0 \leq k \leq \frac{K}{2}-1, \\ r_{\mathrm{PS}}(n, k) & \text { for } \frac{K}{2} \leq k \leq K-1,\end{cases}
$$

where the subscript PS denotes the received signal during the pilot mode. The resulting vector $\mathbf{y}(n)$ is simply the cyclic extension of the received signal after the removal of the guard interval. The vector $\mathbf{y}(n)$ is then demodulated with a DFT to yield the input signal $\mathbf{Y}(n)$ to the channel estimator. With the pilot symbols constructed as in (14), the cyclic property is ensured during the pilot mode and each symbol in $\mathrm{Y}(n)$ contains only the pilot contribution from one transmitter. The complex gain of the $(k+2(m-1))$ th subcarrier from the $m$ th transmitter can be estimated by

$$
\begin{aligned}
& \tilde{\Lambda}_{m}(n, k+2(m-1)) \\
& \quad= \begin{cases}\frac{Y(n, k+2(m-1))}{\operatorname{PS}_{m}(n, k+2(m-1))} & \text { if }(k)_{2 M}=0, \\
0 & \text { otherwise. }\end{cases}
\end{aligned}
$$

Notice that the nonzero estimate

$$
\begin{aligned}
& \tilde{\Lambda}_{m}(n, k+2(m-1)) \\
& \quad=\Lambda_{m}(n, k+2(m-1))+W(n, k+2(m-1)),
\end{aligned}
$$

where $\Lambda_{m}(n, k+2(m-1))$ is the actual complex gain of the $(k+2(m-1))$ th subcarrier from the $m$ th transmitter and
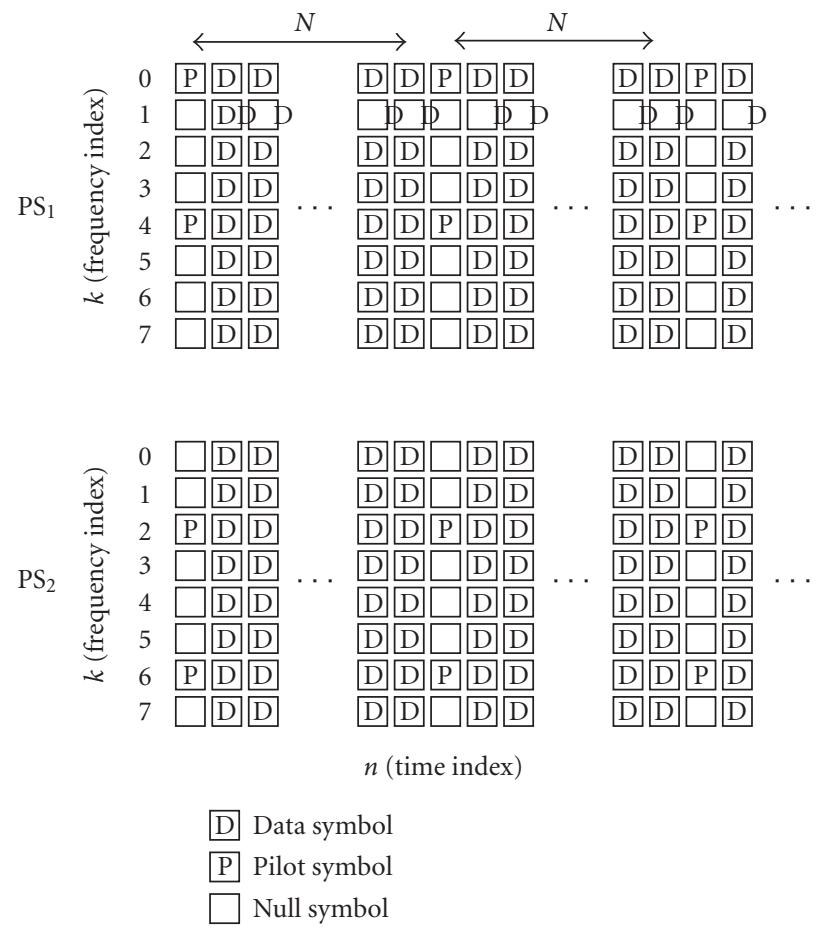

FIgure 9: Pilot symbol patterns for an OFDM transmitter diversity system without a cyclic prefix where $K=8$ and $M=2$.

$W(n, k+2(m-1))$ is the sampled channel noise which is a zero-mean complex Gaussian random variable with variance $\sigma_{W}^{2}=\sigma_{Z}^{2} /(2 M)[27]$.

The diagonal elements of $\tilde{\Lambda}_{m}(n)$ are, in effect, samples of the frequency response of the channel between the $m$ th transmitter and the receiver. Let $\widetilde{\mathbf{h}}_{m}(n)$ be the IDFT of the diagonal of $\tilde{\Lambda}_{m}(n)$. In the absence of noise, $\tilde{\mathbf{h}}_{m}(n)$ is related to the actual CIR $\mathbf{h}_{m}(n)$ by [27]

$$
\tilde{h}_{m}(n, k)=\frac{1}{2 M} \sum_{l=0}^{2 M-1} h_{m}\left(n,\left(k+\frac{K}{2 M} l\right)_{K}\right) e^{j(\pi m / M) l} .
$$

Notice that $\widetilde{\mathbf{h}}_{m}(n)$ is the sum of circularly shifted images of $\mathbf{h}_{m}(n)$. The images in (18) are the direct result of sampling in the frequency domain. To avoid aliasing in the time domain, the condition $K \geq 2 M(L+1)$ must be satisfied. To remove the images, $\tilde{\mathbf{h}}_{m}(n)$ is passed through a length $L+1$ rectangular window of gain $M$ to yield the temporal estimate $\hat{\mathbf{h}}_{m}(n)$ at the pilot instant as follows:

$$
\hat{h}_{m}(n, k)= \begin{cases}h_{m}(n, k)+\xi(n, k), & 0 \leq k \leq L, \\ 0, & L+1 \leq k \leq K-1 .\end{cases}
$$

The DFT of $\hat{\mathbf{h}}_{m}(n)$ yields the estimate of the channel parameters

$$
\widehat{\boldsymbol{\Lambda}}_{m}(n)=\boldsymbol{\Lambda}_{m}(n)+\boldsymbol{\Xi}(n),
$$

where the elements of the noise vector $\Xi(n)$ have a variance of $\sigma_{W}^{2}(2 M(L+1) / K)$. Since $2 M(L+1)<K$ in general, in addition 

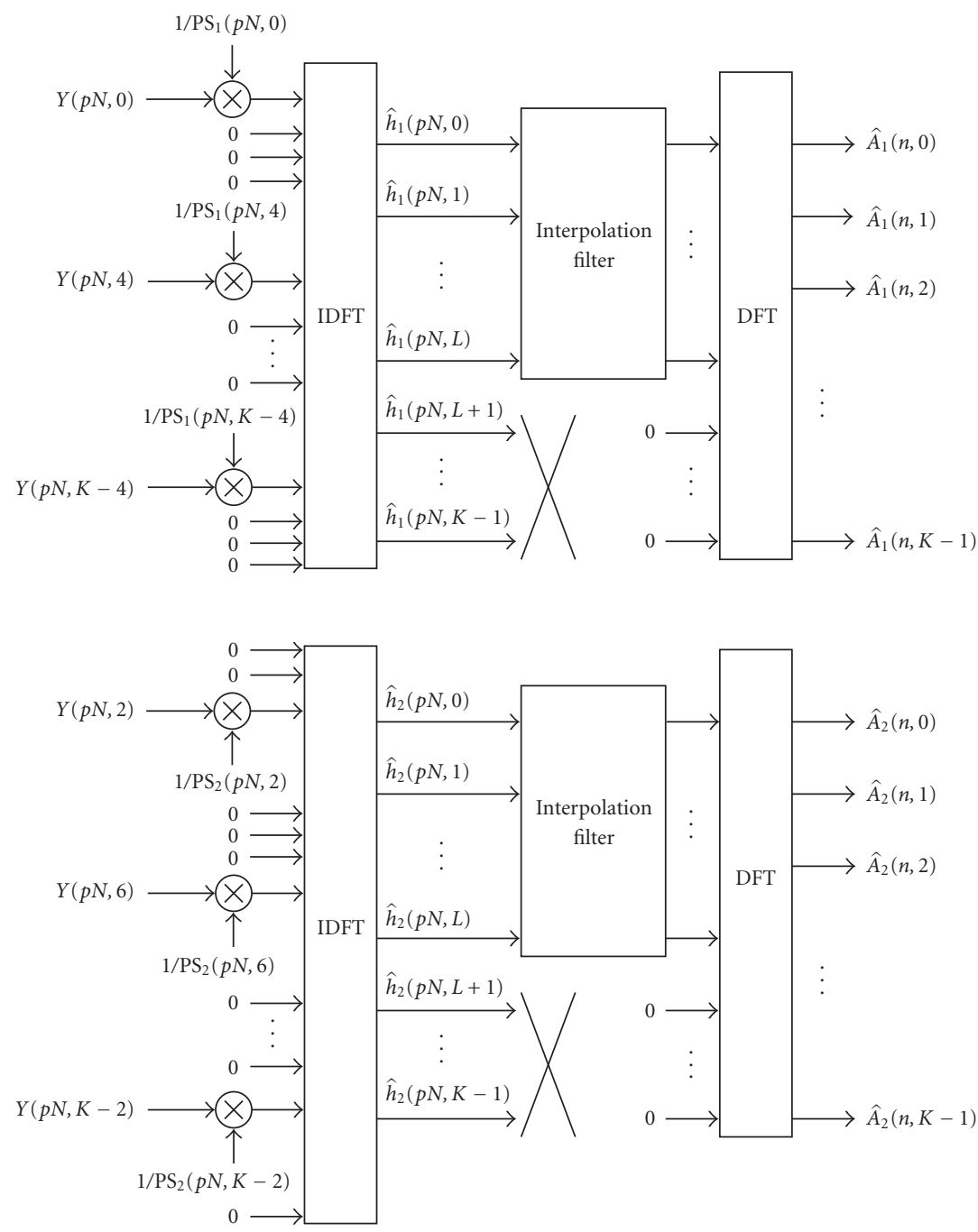

FIGURE 10: Block diagram of the proposed PSA channel estimator for a two-branch OFDM transmitter diversity system without a cyclic prefix.

to removing the images, the windowing operation also reduces the variance of the noise by a factor of $2 M(L+1) / K$. These temporal estimates at the pilot instants $\hat{\mathbf{h}}_{m}(n)$ are then passed through a third-order least-square interpolation filter [26] to provide the estimated channel parameters during the data transmission mode. A block diagram of the proposed PSA channel estimator for a two-branch OFDM transmitter diversity system without a cyclic prefix is shown in Figure 10.

The performance of the proposed PSA channel estimator for OFDM transmitter diversity systems without a cyclic prefix has been evaluated by simulations. The simulations used $K=128$ and $N=20$ for ISTBC-OFDM and $K=256$ and $N=10$ for ISFBC-OFDM. Simulation results of the average BER after two iterations $(i=2)$ for a two-branch ISTBCOFDM system with ideal channel parameters and with channel parameters estimated by the proposed PSA channel estimator with a third-order least-square interpolator are shown in Figure 11. Simulation results for the ISFBC-OFDM system are shown in Figure 12.
At low SNR and with estimated channel parameters, both the ISTBC-OFDM and the ISFBC-OFDM systems have about $2 \mathrm{~dB}$ performance degradation from the corresponding systems using ideal channel parameters. At high SNR, the BER performance of the ISTBC-OFDM system with estimated parameters approaches that with the ideal parameters. The ISFBC-OFDM system, however, still exhibits a slight degradation with estimated parameters, especially in faster fading environments $\left(f_{D}=100 \mathrm{~Hz}\right)$. The ISFBC-OFDM system seems to be more sensitive to channel estimation error at faster fading environments than the ISTBC-OFDM system. The cause of this difference in sensitivity to channel estimation between the two systems is under investigation.

\section{SUMMARY}

Bandwidth efficient ISTBC-OFDM and ISFBC-OFDM transmitter diversity systems have been presented in this paper. A low-complexity PSA channel estimator for OFDM 


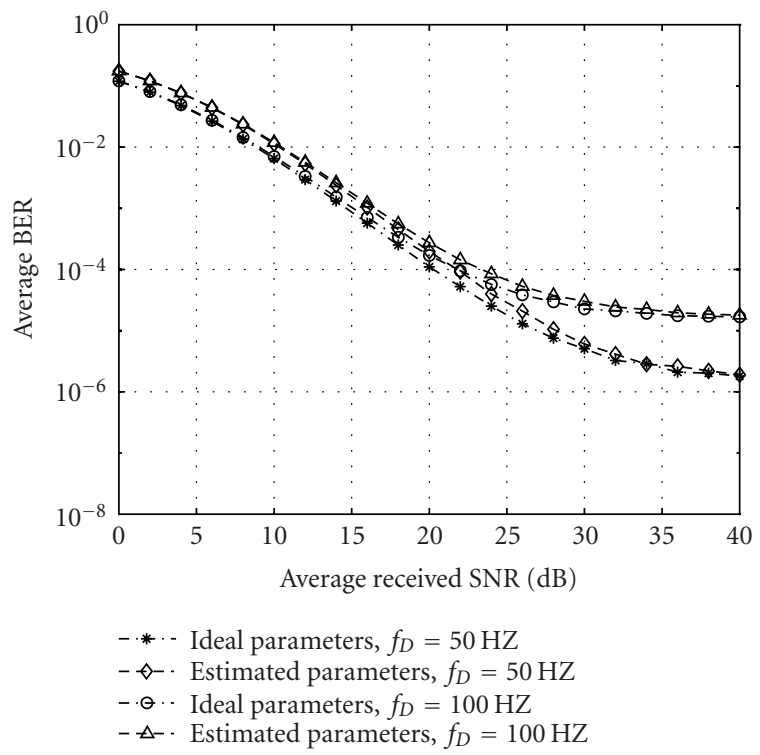

FIGURE 11: Performance comparison of ISTBC-OFDM systems with ideal channel parameters and channel parameters estimated with a third-order least-square interpolator.

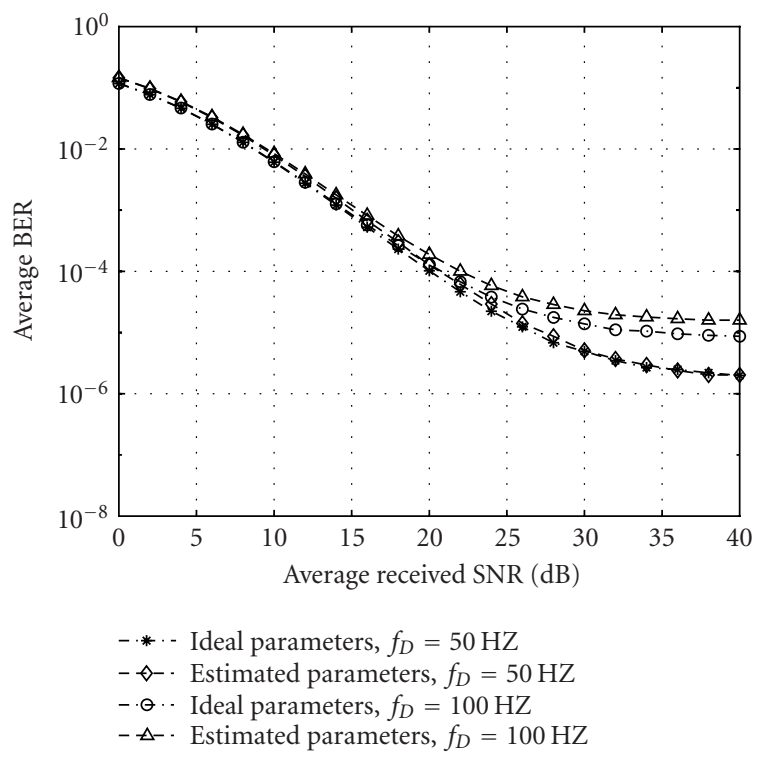

FIGURE 12: Performance comparison of ISFBC-OFDM systems with ideal channel parameters and channel parameters estimated with a third-order least-square interpolator.

transmitter diversity systems without a cyclic prefix, therefore applicable to ISTBC-OFDM and ISFBC-OFDM systems, has also been presented. The proposed ISTBC-OFDM and ISFBC-OFDM systems are shown to be effective and efficient means of eliminating the need for a cyclic prefix while still providing good spatial diversity gain. The computational complexity of the ISTBC-OFDM and ISFBC-OFDM algorithms has been analyzed and shown to be significantly more efficient than other equalization techniques for OFDM sys- tems without a cyclic prefix. More importantly, the ISTBCOFDM and ISFBC-OFDM algorithms are the only known techniques applicable to OFDM transmitter diversity systems without a cyclic prefix.

For ease of presentation, this paper has focused on systems with two transmit antennas $(M=2)$ and a single receive antenna. It should be noted that the proposed approach is also applicable to systems with a larger number of transmit antennas $(M>2)$ and can be easily extended to systems with multiple receive antennas by replicating the proposed technique at each receive antenna branch together with a signal combining scheme similar to that shown in [28, Section III-B].

We have developed the ISTBC-OFDM and ISFBCOFDM algorithms to take advantage of the relatively simple space-time block coding process in the STBC-OFDM and SFBC-OFDM systems. Other more sophisticated OFDM transmitter diversity systems $[29,30,31,32,33]$ have the potential of achieving higher performance than STBC-OFDM and SFBC-OFDM systems, albeit at higher computational complexities. Future work will include applying tail cancellation and cyclic reconstruction techniques to these more recently proposed OFDM transmitter diversity systems for removal of the cyclic prefix and, subsequently, improvement in bandwidth efficiency.

\section{ACKNOWLEDGMENT}

This paper was presented in part at the IEEE International Conference on Acoustics, Speech, and Signal Processing (ICASSP), Orlando, Florida, May 2002.

\section{REFERENCES}

[1] D. Agrawal, V. Tarokh, A. Naguib, and N. Seshadri, "Spacetime coded OFDM for high data-rate wireless communication over wideband channels," in Proc. IEEE Vehicular Technology Conference (VTC '98), vol. 3, pp. 2232-2236, Ottawa, Ont, Canada, May 1998.

[2] Y. Li, J. C. Chuang, and N. R. Sollenberger, "Transmitter diversity for OFDM systems and its impact on high-rate data wireless networks," IEEE Journal on Selected Areas in Communications, vol. 17, no. 7, pp. 1233-1243, 1999.

[3] K. F. Lee and D. B. Williams, "A space-time coded transmitter diversity technique for frequency selective fading channels," in Proc. IEEE Sensor Array and Multichannel Signal Processing Workshop (SAM '00), pp. 149-152, Cambridge, Mass, USA, March 2000.

[4] K. F. Lee and D. B. Williams, "A Space-frequency transmitter diversity technique for OFDM systems," in Proc. IEEE Global Telecommunications Conference (GLOBECOM '00), vol. 3, pp. 1473-1477, San Francisco, Calif, USA, November-December 2000.

[5] A. Ruiz, J. M. Cioffi, and S. Kasturia, "Discrete multiple tone modulation with coset coding for the spectrally shaped channel," IEEE Trans. Communications, vol. 40, no. 6, pp. 10121029, 1992.

[6] R. van Nee and R. Prasad, OFDM for Wireless Multimedia Communications, Artech House, Boston, Mass, USA, 2000.

[7] Commission of European Communities, Digital Land Mobile Radio Communications - COST 207, Office for Official Publications of the European Communities, Luxembourg, 1989. 
[8] N. Al-Dhahir and J. M. Cioffi, "Optimum finite-length equalization for multicarrier transceivers," IEEE Trans. Communications, vol. 44, no. 1, pp. 56-64, 1996.

[9] P. J. W. Melsa, R. C. Younce, and C. E. Rohrs, "Impulse response shortening for discrete multitone transceivers," IEEE Trans. Communications, vol. 44, no. 12, pp. 1662-1672, 1996.

[10] L. Vandendorpe, "MMSE equalizers for multitone systems without guard time," in Proc. 8th European Signal Processing Conference (EUSIPCO '96), vol. 3, pp. 2049-2052, Trieste, Italy, September 1996.

[11] L. Vandendorpe, "Fractionally spaced linear and DF MIMO equalizers for multitone systems without guard time," Annals of Telecommunications, vol. 52, no. 1-2, pp. 21-30, 1997.

[12] Y. Sun and L. Tong, "Channel equalization for wireless OFDM systems with ICI and ISI," in Proc. IEEE International Conference on Communications (ICC '99), vol. 1, pp. 182-186, Vancouver, BC, Canada, June 1999.

[13] J. M. Cioffi and J. A. C. Bingham, "A data-driven multitone echo canceller," IEEE Trans. Communications, vol. 42, no. 10, pp. 2853-2869, 1994.

[14] D. Kim and G. L. Stüber, "Residual ISI cancellation for OFDM with applications to HDTV broadcasting," IEEE Journal on Selected Areas in Communications, vol. 16, no. 8, pp. 1590 1599, 1998.

[15] A. V. Oppenheim and R. W. Schafer, Discrete-Time Signal Processing, Prentice-Hall, Englewood Cliffs, NJ, USA, 1989.

[16] G. H. Golub and C. F. Van Loan, Matrix Computations, Johns Hopkins University Press, Baltimore, Md, USA, 3rd edition, 1996.

[17] G. E. Moore, "Cramming more components onto integrated circuits," Electronics Magazine, vol. 38, no. 8, pp. 114-117, 1965.

[18] J.-J. van de Beek, O. Edfors, M. Sandell, S. K. Wilson, and P. O. Börjesson, "On channel estimation in OFDM system," in Proc. IEEE Vehicular Technology Conference (VTC '95), vol. 2, pp. 815-819, Chicago, Ill, USA, July 1995.

[19] P. Hoeher, S. Kaiser, and P. Robertson, "Two-dimensional pilot-symbol-aided channel estimation by Wiener filtering," in Proc. IEEE Int. Conf. Acoustics, Speech, Signal Processing (ICASSP '97), vol. 3, pp. 1845-1848, Munich, Germany, April 1997.

[20] P. Hoeher, S. Kaiser, and P. Robertson, "Pilot-symbol-aided channel estimation in time and frequency," in Proc. IEEE Global Telecommunications Conference (GLOBECOM '97), Communication Theory Mini-Conference, pp. 90-96, Phoenix, Ariz, USA, November 1997.

[21] O. Edfors, M. Sandell, J.-J. van de Beek, S. K. Wilson, and P. O. Börjesson, "OFDM channel estimation by singular value decomposition," IEEE Trans. Communications, vol. 46, no. 7, pp. 931-939, 1998.

[22] O. Edfors, M. Sandell, J.-J. van de Beek, S. K. Wilson, and P. O. Börjesson, "Analysis of DFT-based channel estimators for OFDM," Wireless Personal Communications, vol. 12, no. 1, pp. 55-70, 2000.

[23] Y. Li, L. J. Cimini Jr., and N. R. Sollenberger, "Robust channel estimation for OFDM systems with rapid dispersive fading channels," IEEE Trans. Communications, vol. 46, no. 7, pp. 902-915, 1998 .

[24] Y. Li, "Pilot-symbol-aided channel estimation for OFDM in wireless systems," IEEE Trans. Vehicular Technology, vol. 49, no. 4, pp. 1207-1215, 2000.

[25] Y. Li, N. Seshadri, and S. Ariyavisitakul, "Channel estimation for OFDM systems with transmitter diversity in mobile wireless channels," IEEE Journal on Selected Areas in Communications, vol. 17, no. 3, pp. 461-471, 1999.

[26] K. F. Lee and D. B. Williams, "Pilot-symbol-assisted channel estimation for space-time coded OFDM systems," EURASIP
Journal on Applied Signal Processing, vol. 2002, no. 5, pp. 507$516,2002$.

[27] N. J. Fliege, Multirate Digital Signal Processing: Multirate Systems, Filter Banks, Wavelets, John Wiley \& Sons, West Sussex, UK, 1994.

[28] S. M. Alamouti, "A simple transmit diversity technique for wireless communications," IEEE Journal on Selected Areas in Communications, vol. 16, no. 8, pp. 1451-1458, 1998.

[29] Y. Gong and K. B. Letaief, "Space-frequency-time coded OFDM for broadband wireless communications," in Proc. IEEE Global Telecommunications Conference (GLOBECOM '01), vol. 1, pp. 519-523, San Antonio, Tex, USA, November 2001.

[30] B. Lu, X. Wang, and K. R. Narayanan, "LDPC-based spacetime coded OFDM systems over correlated fading channels: Performance analysis and receiver design," IEEE Trans. Communications, vol. 50, no. 1, pp. 74-88, 2002.

[31] Y. G. Li, J. H. Winters, and N. R. Sollenberger, "MIMOOFDM for wireless communications: signal detection with enhanced channel estimation," IEEE Trans. Communications, vol. 50, no. 9, pp. 1471-1477, 2002.

[32] A. F. Molisch, M. Z. Win, and J. H. Winters, "Space-timefrequency (STF) coding for MIMO-OFDM systems," IEEE Communications Letters, vol. 6, no. 9, pp. 370-372, 2002.

[33] Z. Liu, Y. Xin, and G. B. Giannakis, "Space-time-frequency coded OFDM over frequency-selective fading channels," IEEE Trans. Signal Processing, vol. 50, no. 10, pp. 2465-2476, 2002.

King F. Lee received his B.S.E.E. degree from the University of Florida, M.S.E. degree from Florida Atlantic University, and Ph.D. degree from the Georgia Institute of Technology. In 1979, he joined Motorola Inc., where he is currently a Distinguished Member of the Technical Staff. His areas of interest include mixed analog-digital integrated circuit design, wireless communications, and digital signal processing. Dr. Lee

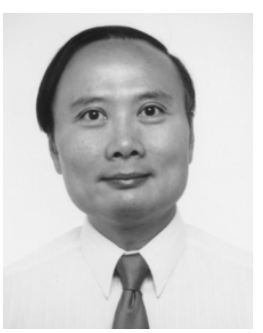
has served as a Member of the Industrial Advisory Board of the NSF Research Center for the Design of Analog-Digital Integrated Circuits (CDADIC) from 1989 to 1993 . He is a Registered Professional Engineer and a Member of the Eta Kappa Nu Honor Society.

Douglas B. Williams received the B.S.E.E., M.S., and Ph.D. degrees in electrical and computer engineering from Rice University, Houston, Texas, in 1984, 1987, and 1989, respectively. In 1989, he joined the faculty of the School of Electrical and Computer Engineering at the Georgia Institute of Technology, Atlanta, Georgia, where he is currently an Associate Professor. There he is also affiliated with the Center for Signal and Image Processing and teaches courses in signal processing and telecommunications. Dr. Williams has served as an Associate Editor of the IEEE Transactions on Signal Processing and is a Member of the IEEE Signal Processing Society's SPTM Technical Committee. He was on the conference committee for the 1996 International Conference on Acoustics, Speech, and Signal Processing that was held in Atlanta and is currently Cochair of the 2002 IEEE DSP and Signal Processing Education Workshops. Dr. Williams was Coeditor of the Digital Signal Processing Handbook published in 1998 by CRC Press and IEEE Press. He is a Member of the Tau Beta Pi, Eta Kappa Nu, and Phi Beta Kappa Honor Societies. 